\title{
Evaluación y Optimización de Secador Hibrido para Yuca
}

\author{
Mauricio J. Sierra y Omar A. Pérez \\ Programa de Ingeniería de Alimentos, Universidad de Córdoba, Km 12 vía a Ciénaga de Oro, Colombia \\ (e-mail: ingalmab@hotmail.com, oaperez@correo.unicordoba.edu.co)
}

Recibido Jul. 29, 2015; Aceptado Sep. 29, 2015; Versión final Nov. 27, 2015, Publicado Abr. 2016

\begin{abstract}
Resumen
El objetivo de esta investigación consistió en establecer los valores óptimos de carga másica, temperatura y velocidad del aire en el secado de yuca, en un secador híbrido, para reducir su contenido de humedad hasta $13 \%$, en base húmeda. Para la evaluación del secador hibrido se desarrollaron corridas experimentales de secado a temperaturas entre 50 y $70^{\circ} \mathrm{C}$, velocidad de aire de 1 a $8 \mathrm{~m} / \mathrm{s}$ y carga másica de 30 a $80 \mathrm{~kg} / \mathrm{m}^{2}$. Para la optimización se empleó el criterio de maximización de la eficiencia global de secado basado en el estudio fenomenológico del proceso. A través de los resultados se delimitó la región factible de optimización por temperaturas entre 50 y $55^{\circ} \mathrm{C}$, velocidad de aire de 1.5 a $1.9 \mathrm{~m} / \mathrm{s}$ y carga másica de 47 a $67 \mathrm{~kg} / \mathrm{m}^{2}$. La máxima eficiencia global de secado obtenida es $65,6 \%$ para temperatura, velocidad de aire y carga másica: $50^{\circ} \mathrm{C}, 1.66 \mathrm{~m} / \mathrm{s}$ y $55.40 \mathrm{~kg} / \mathrm{m}^{2}$ respectivamente, empleado un tiempo de operación de 5.5 horas.
\end{abstract}

Palabras clave: optimización de secado; eficiencia global; yuca; variables óptimas de operación

\section{Evaluation and Optimization of Hybrid Dryer of Cassava}

\begin{abstract}
The objective of this research was to establish the optimal values of mass loading, temperature and air velocity for the drying of cassava, in a hybrid dryer, to reduce its moisture content to $13 \%$ wet basis. For the evaluation of the hybrid dryer, drying experimental runs were carried out at temperatures between 50 and $70{ }^{\circ} \mathrm{C}$, air velocity between 1 and $8 \mathrm{~m} / \mathrm{s}$ and mass loading of 30 to $80 \mathrm{~kg} / \mathrm{m}^{2}$. The optimization criterion was the maximization of the overall efficiency of drying based on the phenomenological study of the process. Through the results, the feasible regions were delimited: for temperatures between 50 and $55^{\circ} \mathrm{C}$, air velocity of 1.5 to $1.9 \mathrm{~m} / \mathrm{s}$ and mass load between 47 and $67 \mathrm{~kg} / \mathrm{m}^{2}$. The maximum overall drying efficiency obtained was $65.6 \%$, for temperature, air velocity and mass loading of $50^{\circ} \mathrm{C}, 1.66 \mathrm{~m} / \mathrm{s}$ and $55.40 \mathrm{~kg} / \mathrm{m}^{2}$ respectively, employing an operating time of 5.5 hours.
\end{abstract}

Keywords: drying optimization; overall efficiency; drying; cassava; optimum operating variables 


\section{INTRODUCCIÓN}

El panorama de la demanda yuca a nivel mundial es de 45 millones de toneladas, una producción reportada hasta el 2013 en Colombia 1.871 .517 toneladas (menor a las producidas en el 2006) (FAO, 2011)), reflejan un el desaprovechamiento de la oportunidades actuales del mercado. La tecnificación del sector yuquero en materia de secado en la costa norte colombiana es bajo, se sigue utilizando herramientas y condiciones de los años 70'S (espesor de capa fija: $12 \mathrm{~cm}$, tiempo de Secado $48-72$ horas, carga máxima: $12 \mathrm{Kg} / \mathrm{m}^{2}$ ) (Lisímaco et al. 2002); situación que no permite afrontar la exigencia actual de cantidad, calidad, tiempo por parte de la industria y futuros incrementos esperados de las áreas sembradas de yuca. A lo largo de 20042013 se han presentado propuestas para modernizar los sistemas de secado de yuca; "secador de yuca, en lecho poroso, usando flujo radial de aire caliente", (Bula et al. 2003); procedimientos basados en el uso de combustibles fósiles, recursos no renovables y de baja eficiencia global (energética), que resultan de poco intereses para los productores y asociaciones de secado de yuca.

A la luz de esta situación, se propuso diseñar, evaluar y optimizar un sistema de secado hibrido a nivel de planta piloto para el programa de ingeniería de alimentos de la Universidad de Córdoba, Colombia, se trabajó en tres etapas: 1) construcción del secador hibrido basado en el protipo de Juarez de Souza et al.(2000) para pequeños caficultores brasileros del estado de Minas de Gerias; 2) evaluación del secador hibrido a través corridas experimentales de secado a condiciones de temperatura y velocidad del aire comprendidas entre 50 - $70^{\circ} \mathrm{C}$ y $1-10 \mathrm{~m} / \mathrm{s}$ respectivamente para una variación de carga másica de yuca entre 30 y $80 \mathrm{~kg} / \mathrm{m}^{2}$ y 3 ) determinación de la interacción optima de las variables independientes antes citadas para obtener un proceso de secado de mayor eficiencia global.

En la literatura se encuentran estudios de diseños de equipos para procesamiento de yuca y en especial para producción de almidón, orientados a la extracción de almidón a partir de trozos secos de yuca (Manihot esculenta Crantz) con mínima utilización de agua (Barona e Isaza, 2003); Evaluación técnica y económica de una planta piloto para la producción de harina integral de yuca para uso en la alimentación animal. (Florián y Rengifo, 2003); Evaluación técnica de la primera etapa de secado en la planta piloto de secado artificial de yuca (Gallego y Tobar, 2003); secador de yuca, en lecho poroso, usando flujo radial de aire caliente (Durango et al. 2004); Optimización energética de un secador radial de yuca" ( Bula et al. 2003); Optimización del sistema mixto de secado de yuca (manihotesculenta crantz.) instalado en la planta agroindustrial de Morroa para corporación Minuto de Dios (Hernández y Vásquez., 2008.) ; Automatización de un secador convectivo de aire caliente para fines de docencia en Ingeniería de Alimentos (Saavedra et al. 2008); Desenvolvimento e avaliação de um secador solar para grãos, associado a silo subterrâneo. (Braga et al. 2005); Estudio del secado de Anacardo (Anacardium occidentale L.) mediante secador solar de radiación directa (Machado et al.2010); Cinética de secado de Musa paradisiaca L. platano y Manihot esculenta Grantz yuca (Carranza y Sánchez, 2002).

\section{MATERIALES Y MÉTODOS}

Determinación de velocidad del aire. La medición de la velocidad de aire superficie del distribuidor, se realizó a través de un anemómetro digital Kestrel 2000, de características.

Determinación de la humedad relativa del aire. La medición de la humedad relativa se determinó a través de un termohigrómetro digital marca HT3600.

Determinación de la radiación solar. La intensidad solar se evaluó a través de un TES 1333 Solor power meter.

Determinación de temperatura del aire. La medición de la temperatura del gas, se efectuó a la entrada de la cámara del sistema de combustión, y en tres puntos en el ducto de distribución de aire caliente. Para realizar esta actividad de empleó termopar digital empotrable D2100.

Determinación de humedad de la yuca fresca y procesada. El procedimiento empleado es el método 950.46/53 de la A.O.A.C ,1997.

Determinación experimental de la curva de secado. El procedimiento abordado consistió en colocar una muestra de yuca húmeda dentro de una canastilla perforada encima del ducto distribuidor del aire del secador hibrido, a distintas relaciones de carga másica: $30,50,65$ y $80 \mathrm{~kg} / \mathrm{m}^{2}$, perpendicular al flujo de aire; a las temperaturas 50, 60 y $70 \stackrel{\circ}{ } \mathrm{C}$ sin interrumpir la operación. Los datos obtenidos de las corridas de secado a las condiciones específicas de velocidad del aire, temperatura y carga másica, se expresaron en base seca como lo relaciona la ecuación (1), se calcularon los datos de peso a cada instante de tiempo de secado $W$ (peso del sólido húmedo en $\mathrm{kg}$ ) y el peso del sólido seco Ws. se determinó el contenido de humedad de equilibrio, $X^{*}$ $\mathrm{Kg}$ de humedad de equilibrio del sólido seco); para calcular el valor del contenido de humedad libre $X l_{\text {ibre }}$ en $\mathrm{Kg}$, para cada valor de $X_{(t)}$ a través de la ecuación (2). Se calcula la velocidad $N x$ para cada punto a través ecuación (3) ( $N x: \mathrm{Kg} \mathrm{H} 2 \mathrm{O} / \mathrm{h}^{*} \mathrm{~m}^{2}$ ) y $A$ es el área superficial expuesta al secado en $\mathrm{m}^{2}$. Para obtener $N x$ se obtuvo graficando en función del contenido de humedad (Geonklopis, 1998). 


$$
\mathrm{X}_{(\mathrm{t})}=\left(\mathrm{W}-\mathrm{W}_{\mathrm{s}}\right)(\mathrm{kg} \text { totales de agua }) / \mathrm{W}_{\mathrm{s}}(\mathrm{kg} \text { de sólido seco })
$$

$$
\begin{aligned}
& X_{\text {libre }}=X_{(t)}-X^{*} \\
& N x=-\left(W_{S} / A\right)^{*}(d X / d t)
\end{aligned}
$$

Eficiencia de la combustión y Eficiencia global del proceso. Para conocer la eficiencia térmica del sistema calentamiento y del proceso, primero se determinó el calor de combustión, poder calórico del combustible (PCS), y de los gases de chimenea QGases y la energía entregada al aire (QDisipado) empleando las ecuaciones (4) y (5), la eficiencia de combustión, $\eta$, se estableció a través se empleó la ecuación (6). El diseño del sistema de calentamiento, los gases de chimenea son aprovechados; circulando en corriente directa con el aire calentado (Serrano y Carranza, 2005). Eficiencia global del proceso se evaluó a través de la ecuación (7), propuesta por Hernández et al. (2013), siguiendo los pasos: 1) Determinación del agua evaporada por unidad de sólido seco; 2) Cálculo del calor total requerido para el secado de la yuca hasta el 13 base húmeda; 3) Determine la eficiencia global del sistema de secado a través de la ecuación (7)

$$
\begin{aligned}
& Q_{\text {Disipado }}=P C S-Q_{\text {Gases }} \\
& Q_{\text {Gases }}=h_{(\text {Prod(temperatura chimenea) }))}-h_{(\text {reactivos }(298,16 \mathrm{~K}))} \\
& N_{\text {global }}=\left(P C S-h_{(\text {Prod(temperatura chimenea }))}-h_{(\text {reactivos }(298,16 \mathrm{~K}))}\right) / P C S \\
& N_{\text {global }}=\left(X_{L_{\text {Libre }}}{ }^{*}\left(\mathrm{~W}_{\mathrm{S}} / \mathrm{A}\right)^{*} \Delta \mathrm{h}_{\mathrm{fg}}\right) /\left(Q_{\text {Disipado }}+\sum Q_{\text {Pérdido }}+\mathrm{W}_{\text {vent }}+Q_{\text {Radiación Solar }}\right)
\end{aligned}
$$

$\mathrm{h}_{(\text {Prod(temperatura chimenea) })}=$ Entalpia de los gases de chimenea evaluada a la temperatura de la salida.

$\mathrm{h}_{(\text {reactivos }(298,16 \mathrm{~K}))}=$ Entalpia del combustible, oxígeno a la temperatura ambiente.

$\Delta \mathrm{h}_{\mathrm{fg}}=$ Calor latente de vaporización del agua

Optimización de secador hibrido. La metodología empleada para optimizar el secador hibrido para yuca, es la propuesta por Himmelblau en su libro Optimization of chemical processe, 2001 que se describe a través de seis pasos: 1) Identificación de las variables de decisión o independiente, a través del estudio fenomenológico del proceso, que describe el problema de optimización del secador; 2 ) Determinar el criterio de optimización, especificar la función objetivo en términos de las variables de decisión. Maximización de $\mathrm{N}_{\text {global }}$; 3 ) Establecimientos de las limitaciones del proceso de secado, en términos expresiones matemáticas de igualdad, desigualad, paramétricas; 4) Delimitación de la región factible, zona donde existen todas las posibles soluciones al problema de optimización; 5) Determinar el método de optimización (método numérico) que conlleve a las especificaciones de los valores óptimos de velocidad, temperatura del aire, espesor del lecho de sólido que hagan $\mathrm{N}_{\text {global }}$ sea máxima.

\section{RESULTADOS Y ANÁLISIS}

Curvas de secado. Los resultados obtenidos pérdida de peso (humedad en base seca) con respecto al tiempo, se encuentran representados en el gráfico 1 para la temperatura de secado de $50^{\circ} \mathrm{C}$, velocidades de aire de 1 a $8 \mathrm{~m} / \mathrm{s}$ y cargas másicas $30,50,60$ y $80 \mathrm{~kg} / \mathrm{m}^{2}$. Las figuras 1 a), b) c) y d) evidencia que el tiempo de secado de los trozos de yuca para las diferentes relación cargas másicas, disminuye a medida que se incrementa la velocidad de circulación del aire a través del lecho, este comportamiento lo describe Carranza y Sánchez (2002) en secado de yuca y plátano; Gómez y Ochoa (2012) en secado de zanahoria con bomba de calor y convección forzada; Mahecha (2012), en secado de uchuva, simulación de secador solar con convección forzada desarrollada por Benhamou, et al. (2014); Motevali y Minaei. (2012) a través effects of microwave pretreatment on the energy and exergy utilization in thin layer drying of sour pomegranate arils; $y$ Ndubisi et al., (2014) con energy and exergy analyses of native cassava starch drying in a tray dryer.

Otro hecho de importancia que exhibierón las curvas de velocidad de secado de la yuca ilustradas en los graficos 2 a) y b)es ausencia del periodo de secado a velocidad constante, predominando el periodo descreciente por difusión como describe Treybal (1988).

Eficiencia global del proceso secado. Este parámetro se evaluó para todos las corridas de los experimentos, resultantes de las interacciones de la velocidad, temperatura y carga másica; para facilitar esta actividad se construyó curvas de secado para cada condición, extrayendo de las mismas el tiempo de secado requerido hasta alcanzar una humedad del $13 \%$ en base húmeda, además de la determinación la pérdida de calor por paredes (ver figuras 2 a) y b) y 3 ), (Lesme et al. (2011). 

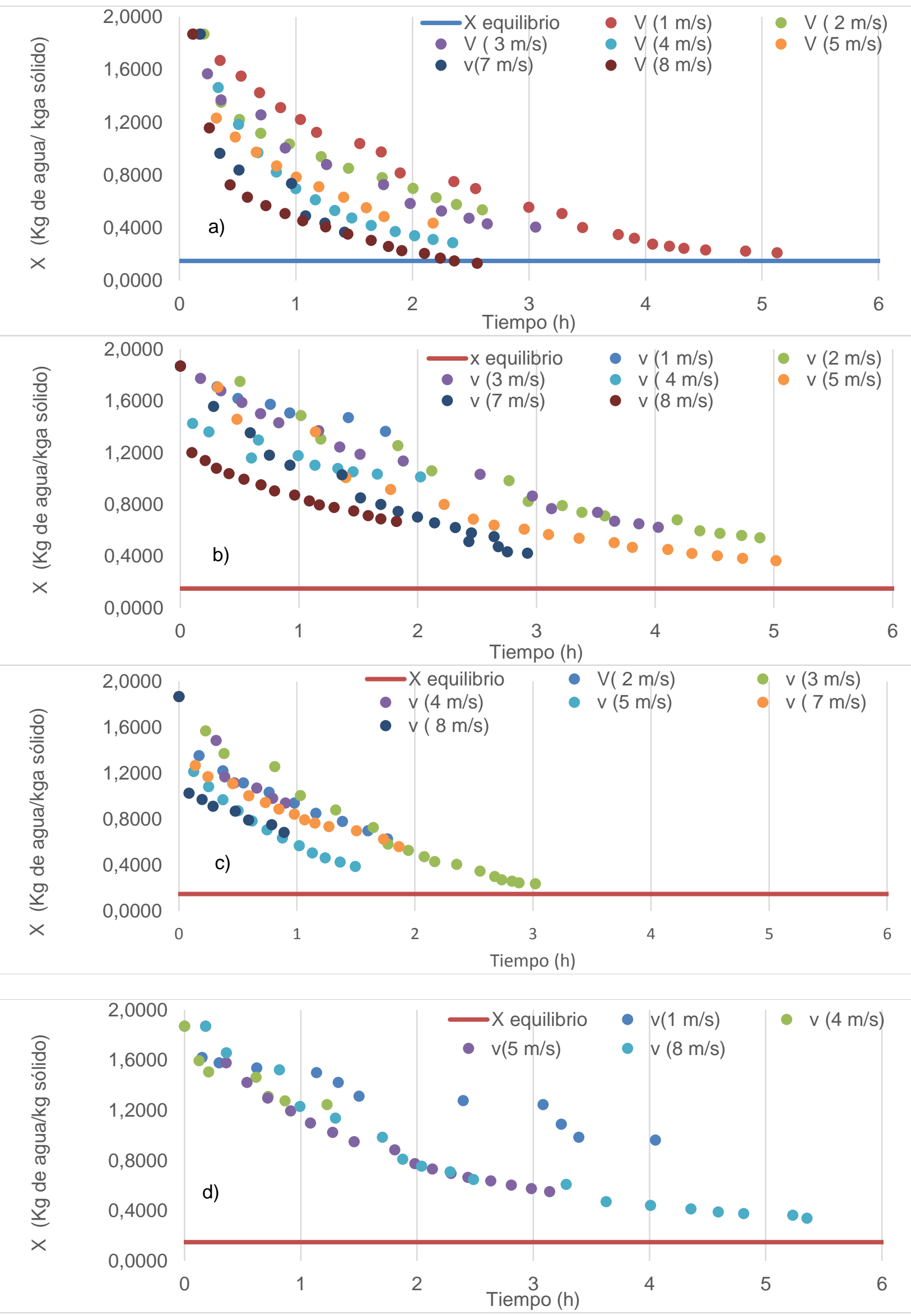

Fig. 1: Curvas de secado a la temperatura de $50^{\circ} \mathrm{C}$ a las velocidades 1 a $8 \mathrm{~m} / \mathrm{s}$ : a) carga másica de $30 \mathrm{~kg} / \mathrm{m}^{2}$, b) carga másica de $50 \mathrm{~kg} / \mathrm{m}^{2} \mathrm{c}$ ) carga másica de $60 \mathrm{~kg} / \mathrm{m}^{2}$, d) carga másica de $80 \mathrm{~kg} / \mathrm{m}^{2}$. 


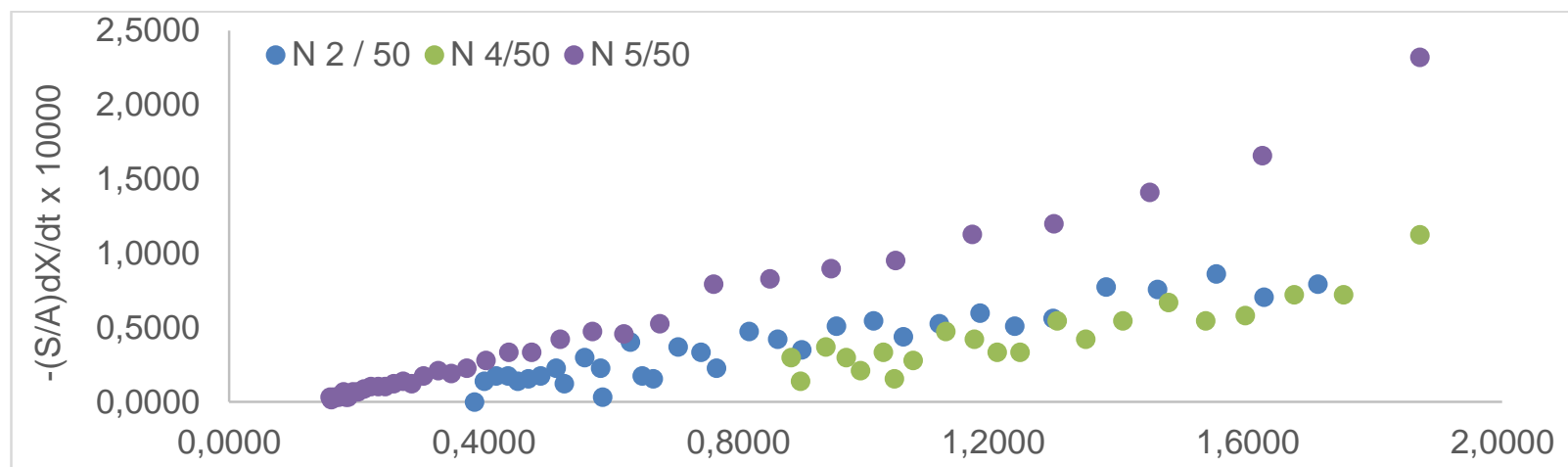

a) X (Kg de agua/kg a sólido)

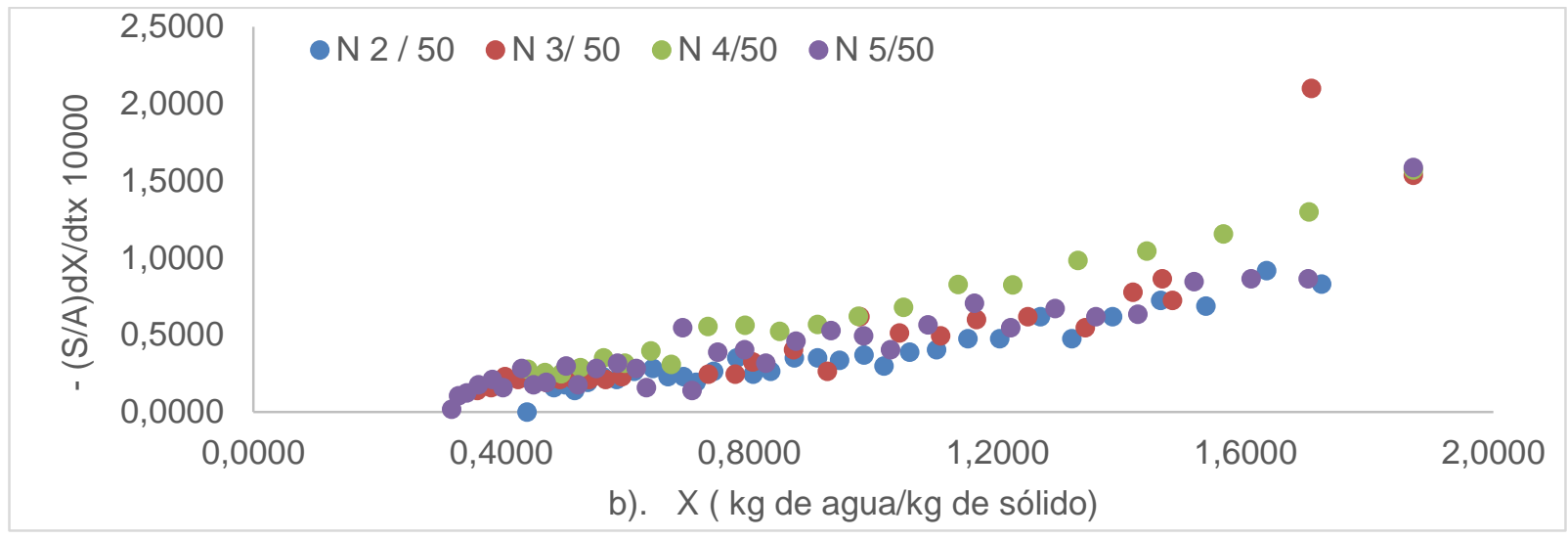

Fig. 2. Curva de velocidad de secado en función humedad en base seca a) $50^{\circ} \mathrm{C}, 50 \mathrm{Kg} / \mathrm{m}^{2}$, velocidad de 2 a $5 \mathrm{~m} / \mathrm{s}$, b) $60^{\circ}, 50 \mathrm{Kg} / \mathrm{m}^{2}$, velocidad de 2 a $5 \mathrm{~m} / \mathrm{s}$

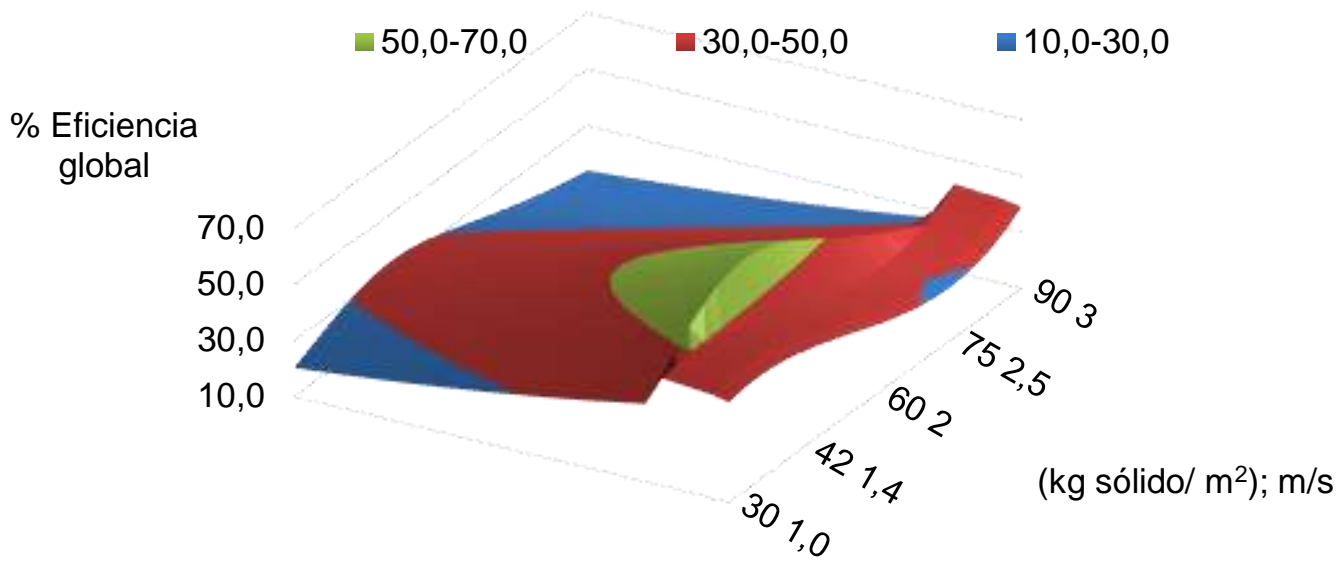

Fig. 3: Curvas de eficiencia de secado a la temperatura de $50-70^{\circ} \mathrm{C}$, carga másica $30-80 \mathrm{~kg} / \mathrm{m}^{2}$, velocidad de $1-4 \mathrm{~m} / \mathrm{s}$, vista superior.

Consumo de combustible. El consumo de combustible se expresó en término de las variables de decisión, para facilitar la búsqueda de los valores óptimos a hiciera la función objetivo sea máxima; para este caso el gasto de gas propano está relacionado con la temperatura y la velocidad del aire de secado. A razón que a medida que exista más masa aire a calentar, mayor ha de ser el flujo de combustible a quemar cuanta más alta sea la temperatura que se quiere alcanzar (ver figura 4). Ahora entendiendo que la combustión es una reacción química se utilizó la expresión de arrhenius para consumo de gas propano relacionando la variable velocidad del aire. El figura 4, muestra una relación directa entre la velocidad del aire y consumo de combustible de la forma $F=Q^{*} V$, donde la pendiente es diferente para cada temperatura y corresponde a la constante de reacción de combustión de gas propano, la cual se modeló como una reacción química de orden uno, $Q=Q_{o}{ }^{*} \exp ^{E a / R T}$ (Da Silva, 2002). El modelo propuesto para explicar el consumo de combustible en 
términos de la velocidad y temperatura del aire de secado es la ecuación (8), cuyos parámetros propios $\mathrm{E}_{a} / \mathrm{R}$, energía de activación y Qo: coeficiente pre-exponencial de consumo de combustible se determinaron a través del método de mínimos cuadrados en términos de la velocidad del aire (1 hasta $8 \mathrm{~m} / \mathrm{s}$ ) y temperatura (50 y $70^{\circ} \mathrm{C}$ ). Modelo propuesto logra explicar en $99.1 \%$ el consumo de gas propano con las variables antes mencionadas: el error promedio asociando en la predicción es de $4.96 \%$.

$$
\begin{aligned}
& \mathrm{Q}=\mathrm{Q}_{0}^{*}\left[\exp ^{\mathrm{Ea} / \mathrm{RT}}\right]^{*} \mathrm{~V} \\
& \mathrm{Q}=45731,675^{\star}[\operatorname{Exp}(-3776,799 / \mathrm{T})]^{*} \mathrm{~V}(\mathrm{Kj} / \mathrm{Kg} \text { aire })
\end{aligned}
$$

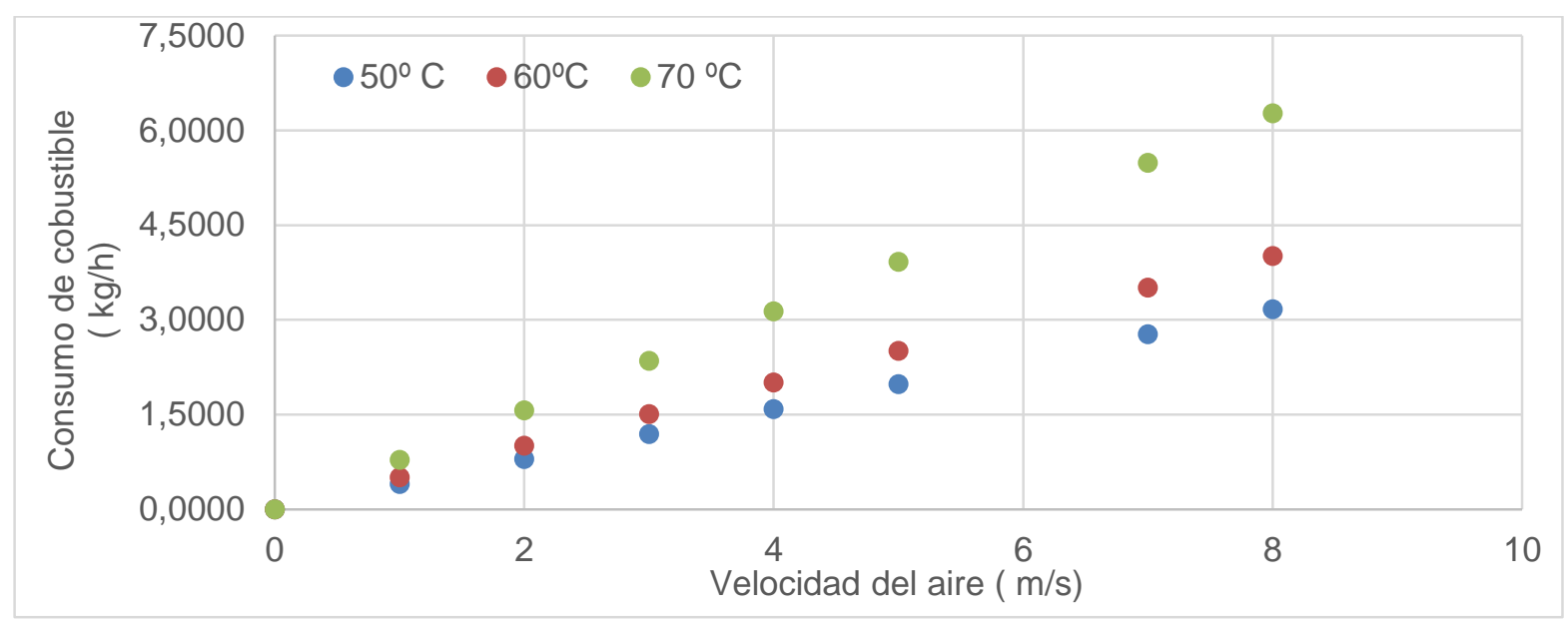

Fig. 4: Consumo de combustible de propano con respecto a la velocidad del aire a las temperaturas 50,60 y $70^{\circ} \mathrm{C}$.

Pérdida de calor a través de las paredes. El calor ganado por el ambiente se determinó a través del análisis de convección natural, este fenómeno está ligado a la temperatura del aire, a la capacidad de aislamiento, área del equipo, condiciones del aire circundante (Cengel, 2007). La ecuación (9), se relaciona con la temperatura de secado a través de las expresiones (10) obtenidas al analizar su comportamiento promedio con respecto a dicha variable.

$$
\begin{aligned}
& \text { QPérdido }=A_{\text {equipo }}{ }^{*} h_{\text {Conv Natural }(T)}{ }^{*}\left(T_{W}(T)-T_{\infty}\right) \\
& \mathrm{T}_{\mathrm{W}(\mathrm{T})}=1,2467^{*} \mathrm{~T}_{\text {Secado }}-9,8339\left[{ }^{\circ} \mathrm{C}\right]
\end{aligned}
$$

Trabajo de sistema de ventilación. El trabajo que desarrolla el sistema de ventilación resulta de multiplicar la potencia del equipo por el tiempo de operación, en este caso el periodo de secado. La variable de decisión involucrada con este parámetro de optimización es la velocidad del aire, la expresión (11).

$$
W_{\text {vent }}=\left(V^{*} A_{\text {circulación }}{ }^{\text {Densidad aire }}{ }^{*} \mathrm{~g}^{*} \mathrm{H}_{\text {Ventilador }}{ }^{*} \text { secado }\right) /\left(1000 \mathrm{n}_{\text {ventilador }}\right)[\mathrm{Kj}]
$$

Tiempo de secado. Esta variable es producto de la interacción de todas condiciones de proceso secado, velocidad del aire, temperatura, carga másica de sólido, de las características del material secar, de la intensidad de radiación solar. El caso estudiado, evidencia un proceso de secado controlado por la difusión como se evidencia en los figuras 2 a) y b); lo que indica que el fenómeno puede reproducirse a través modelo mono-dimensional de una lámina infinita (Arranz et al. 2011). Las ecuaciones (12) y (13) definen el proceso:

$$
\begin{aligned}
& \mathrm{M}(\mathrm{x}, \mathrm{t}=0)=\mathrm{Mo} \\
& \mathrm{dm} / \mathrm{dt}=\mathrm{Dm}^{*}\left(\mathrm{~d}^{2} \mathrm{M} / \mathrm{dx}^{2}\right) \quad \mathrm{Dm}^{*}(\mathrm{dM} / \mathrm{dX})_{\mathrm{x}=0}=0 \\
& -\mathrm{Dm}^{*}(\mathrm{dM} / \mathrm{dX})=\mathrm{Ka}^{*}\left(\mathrm{M}_{(\mathrm{t})}-\mathrm{M}_{\text {Equilibrio }}\right) \\
& \left(M_{(x, t)}-M o\right) /\left(M o-M E_{\text {quilibrio }}\right)=\sum\left(2^{*} \sin \left(U_{n}\right)^{*} \cos \left(U_{n}{ }^{*} X / L\right) \operatorname{EXP}\left(-U^{2} n^{*}\left(D x t / L^{2}\right)\right) /\left(U n+\sin (U n)^{*} \cos (U n)\right)\right.
\end{aligned}
$$

Al realizar la evaluación del secado difusional se encontró que no se explica el comportamiento tiempo de secado para las diferentes condiciones de velocidad y temperatura, por lo tanto el modelo descrito con la 
ecuación (13); no se puede aplicar como modelo de predicción para las variables de este estudio, a consecuencia de la formación decanales en el lecho de yuca como lo indica figura 5.

$$
\begin{aligned}
& t_{\text {secado }}=a(v){ }^{*} C^{2}+b_{(v)}{ }^{*} C+d_{(v)} \\
& a_{(v)}=a_{(0)}{ }^{*} V^{3}+a_{(1)}{ }^{*} V^{2}+a_{(2)} * V+a_{(3)} \\
& b_{(v)}=b_{(0)}{ }^{*} V^{3}+b_{(1)}{ }^{*} V^{2}+b_{(2)}{ }^{*} V_{+} b_{(3)} \\
& d_{(v)}=d_{(0)}{ }^{*} V^{3}+d_{(1)}{ }^{*} V^{2}+d_{(2)}{ }^{*} V+d_{(3)} \\
& t_{\text {secado }}=\left(a_{(0)}{ }^{*} V^{3}+a_{(1)}{ }^{*} V^{2}+a_{(2)}{ }^{*} V+a_{(3)}\right)^{*} C^{2}+\left(b_{(0)}{ }^{*} V^{3}+b_{(1)}{ }^{*} V^{2}+b_{(2)}{ }^{*} V+b_{(3)}\right)^{*} C+\left(d_{(0)}{ }^{*} V^{3}+d_{(1)}{ }^{*} V^{2}+\right. \\
& \left.\mathrm{d}(2)^{\star} \mathrm{V}+\mathrm{d}(3)\right)
\end{aligned}
$$

Tabla 1: Parámetros de la ecuación (16)

\begin{tabular}{|l|r|l|r|l|r|}
\hline$a_{(0)}$ & $-1,3889 E-05$ & $b_{(0)}$ & $-5,1389 E-03$ & $d_{(0)}$ & $3,0833 E-01$ \\
\hline$a_{(1)}$ & $6,2500 E-04$ & $b_{(1)}$ & $-8,7500 E-03$ & $d_{(1)}$ & $-1,3000 E+00$ \\
\hline$a_{(2)}$ & $-4,4611 E-03$ & $b_{(2)}$ & $3,2939 E-01$ & $d_{(2)}$ & $-5,4083 E+00$ \\
\hline$a_{(3)}$ & $1,1883 E-02$ & $b_{(3)}$ & $-1,0882 E+00$ & $d_{(3)}$ & $3,0500 E+01$ \\
\hline
\end{tabular}

Con respecto a lo anterior, se decidió evaluar el comportamiento del tiempo de secado con respecto a la velocidad y carga másica de sólido, con el objeto de identificar un patrón repetitivo. Se graficó los diferentes tiempos de secado en función de la carga de la carga másica y cada parámetro de la ecuación (14) se expresó en términos de la velocidad de aire representado por la ecuación (15). Reemplazando (15) en (14), obtenemos (16). Los coeficientes de la ecuación (16), registrados en la tabla 1, se determinaron a través del método de mínimos cuadrados, Los resultados comparativos entre los tiempos de secado reales y los predichos, describen que el modelo semiempirico propuesto explica un $92,9463 \%$ de la variabilidad del tiempo de respuesta con un margen de error en el cálculo $10,2386 \%$.

Definición del problema de optimización del secador. Una vez expresados todos los elementos que definen la función objetivo en términos de las variables de decisión, se procedió a definir el problema, establecimientos las limitaciones del proceso de secado, es decir las restricciones, actividad que enmarca la región factible donde existen todas las posibles soluciones de optimización. Las restricciones planteadas para este problema de optimización son dos, una para un tiempo de secado menor a igual a 5 horas, a razón que la radiación solar tiene una duración por día de 8 a 10 horas en Berástegui corregimiento de Ciénaga de Oro, Colombia, lo que permitiría obtener al menos dos Bach de producción, periodo que está dentro la jornada laboral normal. La otra restricción es la pérdida de calor a través de las superficies del secador inferior o igual al $20 \%$ del combustible quemado. Los métodos de optimización empleados para establecer los valores óptimos de velocidad, temperatura del aire y carga másica de sólidos para hacer $\mathrm{n}_{\text {global }}$ máxima, fueron Newton -Rapson, estimación cuadráticas y derivadas centrales a través de paquete ofimático Solver de Excel 2007 de Microsoft office.

La tabla 2 relaciona la región factible y las predichas respectivamente, los valores óptimos de carga másica de sólidos, temperatura y velocidad del aire de secado encontrados a través de la modelación del fenómeno de secado hibrido son $55,3849 \mathrm{~kg} / \mathrm{m}^{2}, 50^{\circ} \mathrm{C}$ y $1,66 \mathrm{~m} / \mathrm{s}$ para una eficiencia global máxima alcanzada de 65,65 \%; comparando con trabajos de investigación en esta línea tenemos a López, 2011 con la caracterización y evaluación de un secador hibrido-gas GLP obtuvo eficiencia global máxima de $60 \%$ de secado para una temperatura de operación entre $50-62^{\circ} \mathrm{C}$; Corzo et al. (2008) alcanzóuna eficiencia global máxima energética en el secado de coroba alrededor de la $1,18 \mathrm{~m} / \mathrm{s}$ de velocidad del aire; Boughali et al. (2009) reporta la cinética de secado realizada en un secador híbrido solar-eléctrico con una cámara de secado de 6 charolas, con una carga de $12 \mathrm{Kg}$ de jitomate y un colector solar de $2.45 \mathrm{~m} 2$ de área con una eficiencia global máxima de secado de 31\%, 37, 4 \% alcanzó Amer, et al. (2010) con un secador hibrido para bananas; Guimarães et al., 2013 a través de un prototipo de secador por convección forzada con un resistencia eléctrica obtuvo una eficiencia global máxima de $18.3 \%$ en secado bananas empleando temperaturas de $50-70^{\circ} \mathrm{C}$, flujo de aire 0.5 a $2 \mathrm{~m} / \mathrm{s}$. 
Tabla 2. Formulación del problema de optimización

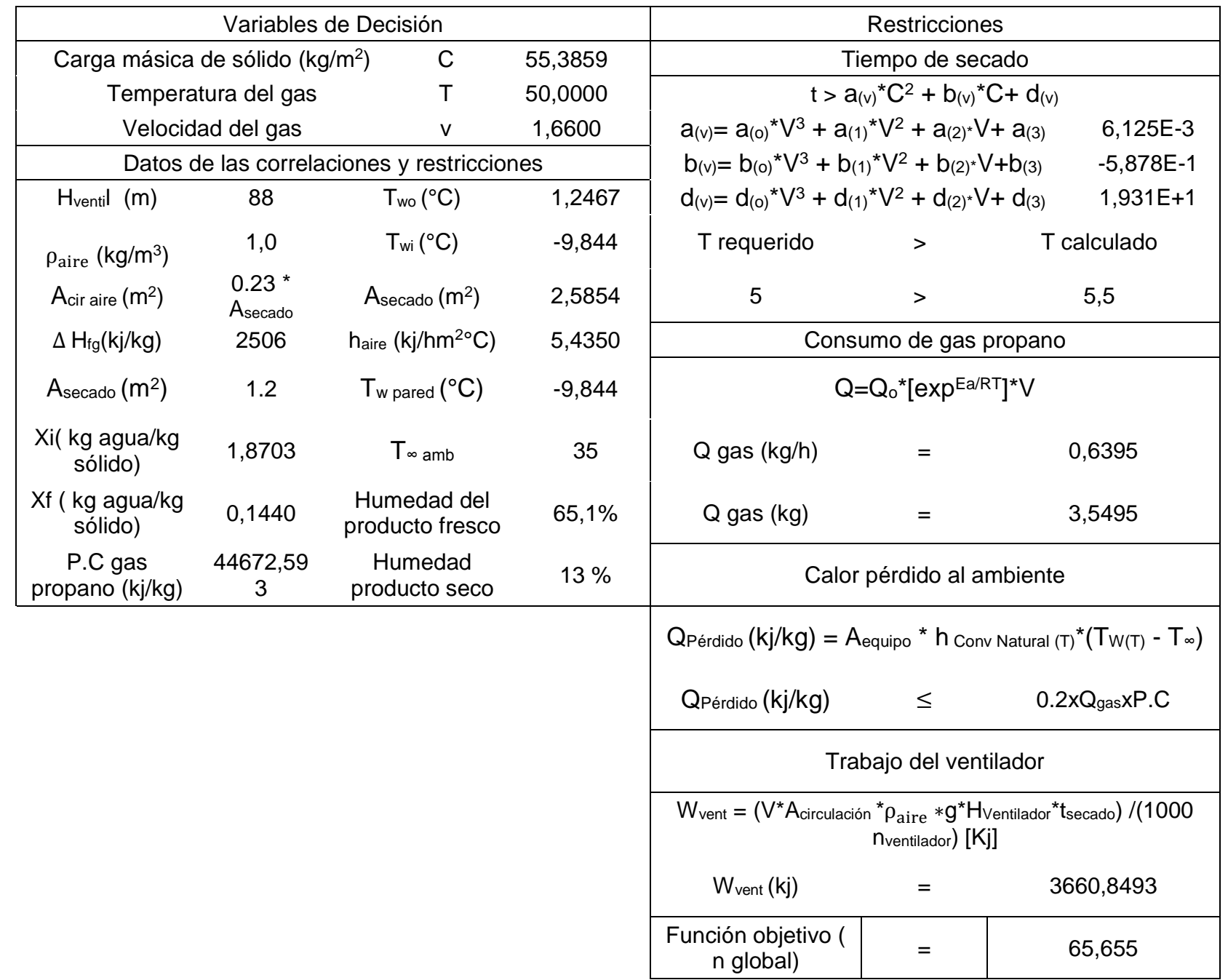

\section{CONCLUSIONES}

1) La región factible del problema de optimización está comprendida para una temperatura de $50^{\circ} \mathrm{C}$, para un rango de velocidad del aire de 1,5 a 1,9 m/s y carga másica de sólido entre 45 y $67 \mathrm{~kg} / \mathrm{m}^{2}$.

2) Las restricciones del problema de optimización con respecto a la perdida de calor del menor o igual al 20 $\%$ de la combustión de gas propano y un tiempo de secado inferior a 5 horas, no ofrece la máxima eficiencia global experimental, así que se incrementó el tiempo de secado a 5.5 horas.

3) La eficiencia global máxima de $65,65 \%$ del proceso de secado hibrido a través de equipo diseñado y construido, para una humedad final $13 \%$ en base húmeda para valores de velocidad, temperatura de aire y carga másica de sólido de: $1,66 \mathrm{~m} / \mathrm{s}, 50^{\circ} \mathrm{C}$ y $55,3847 \mathrm{~kg} / \mathrm{m}^{2}$ respectivamente.

4) Las condiciones óptimas encontradas a través de esta investigación permite operar con una carga de sólidos 3 veces mayor a los sistemas tradicionales de secado de yuca empleando tiempo de operación menor de 7 horas como lo reporta Lisímaco et al. (2002).

\section{REFERENCIAS}

Amer, B; Hossain, M; Gottschalk, K. doi:10.1016/j.enconman.2009.11.016. Design and performance evaluation of a new hybrid solar dryer for banana.(en línea), Energy Conversion and Management 51813 820. (2010)

AOAC. Assocciation of Oficial Analytical Chemist. Oficial methods of análisis. 16 Ed. AOAC , 3005 (1997)

Arranz, F., Correa, E., Jimenez, h., Diezma, B., Garcia-Hierro, J., Robia, J., y otros. Empleo de métodos númericos para el ajuste de los coeficientes de difusividad y convectivo de transferencia de masa en el secado de alimentos. VI Coongreso Ibérico de Agroingeniería , 1-8 (2011) 
Aviara, N; Onuoha, L; Falola, O; Igbeka, J. Energy and exergy analyses of native cassava starch drying in a tray dryer. http://dx.doi.org/10.1016/j.energy.2014.06.087. Energy 73. 809-817 (2014)

Barona R., S., y Isaza, G. Estudios para el desarrollo de un proceso de extracción de almidón a partir de trozos secos de yuca (Manihot esculenta Crantz) con mínima utilización de agua. Cali. Colombia: Universidad Nacional de Colombia (2003)

Benhamou, A;Fazouane, F; Benyoucef, B. Simulation of solar dryer performances with forced convection experimentally proved. doi: 10.1016/j.phpro.2014.07.015.. Physics Procedia (55) 96 - 105 (2014)

Boughali, S. Crop drying by indirect active hybrid solar - Electrical dryer in the eastern Algerian Septentrional Sahara". Solar Energy, Vol. 83, 2223-2232, (2009)

Braga, C. A. Desenvolvimento e avaliação de um secador solar para grãos, associado a silo subterrâneo. . Revista Brasileira de Engenharia Agrícola e Ambiental, 9(4) ,666-670 (2005)

Bula, N. D. Optimización energética de un secador radial de yuca. Ingeniería y Desarrollo.l(14) , I (14), 24-37 (2003)

Carranza, J., y Sanchez, M. Cinética de secado de Musa paradisiaca L. platano y Manihot esculenta Grantz yuca. revista Amazónica de investigación , 15-25 (2002)

Cengel, Y. Transferencia de calor y masa. New York: Mc Graw Hill (2007)

Corzo, O;Bracho, N; Vásquez; A; Pereira, A. Energy and exergy analyses of thin layer drying of coroba slices. doi:10.1016/j.jfoodeng.2007.05.008. Journal of Food Engineering 86, 151-161 (2008)

Da Silva, L. Combustao de propano en leitos fluidizados de areia. Praca Gomes Teixeira: niversidade Do Porto ( 2002)

Durango N., B. A. Construcción de un modelo de secador de yuca, en medio poroso, usando flujo radial de aire caliente. Ingeniería y Desarrollo. I(15) , 9-30 (2004)

Himmelblau, E y Lasdon, L. Optimization of chemical processes. New York: $2001-651(2001)$

FAO. Perpectivas alimentarias, Análisis de los mercados internacionales. Roma: FAOSTAT (2011)

Florián N., R., y Rengifo V., P. Evaluación técnica y económica de una planta piloto para la producción de harina integral de yuca para uso en la alimentación animal. . Cali: Universidad Nacional de Colombia Sede Palmira (2003)

Gallego C., S., y Tobar R., L. Evaluación técnica de la primera etapa de secado en la planta piloto de secado artificial de yuca, CLAYUCA-Protón. . Cali.: Universidad del Valle (2003)

Geonklopis, C. Procesos de transporte y operaciones unitarias 3ed. CUERNA VACA, MEXICO: CECSA (1998)

Gomez, J., y Ochoa, C. aspectos cinéticos y termodinámicos del secado asistido por bomba de calor y el secado convectivo con recirculación total . Seminario Internacional de secado agrícolas , 3-8 (2012)

Guimarães, A; Pereira, L; Brasil, C; Mattedi, J; Torres, A. Tecnnical feasibility assesment of an electrical driyer for banana drying. http://www.abcm.org.br/anais/cobem/2013/PDF/612.pdf. ISSN 2176-5480. 22nd International Congress of Mechanical Engineering (COBEM 2013) November 3-7, 2013, Ribeirão Preto, SP, Brazil, (2013)

Hernández, A., y Vasquez, L. Optimización del sistema mixto de secado de yuca (manihotesculenta crantz.) instalado en la planta agroindustrial de Morroa para corporación Minuto de Dios. Sincelejo.Colombia:

Unversidad de Sucre (2008)

Hernandez-Díaz, W., Hernández, F., Vargas, Z., y Rodriguez, G. y. Coffe grain rotary drying optimization. Revista mexicana de Ingeniería Química, 12(2) , 1-11 (2013)

Juarez de Souza y Silva, M. C. Manual de construçäo e manejo de terreiros para secagem de café. Engeharia Na Agricultura, I(11) , 1-25 (2000) 
Lesme, R., Martinez, J., y Viera, R. S. Evaluación teorica expeimental de un sistema avanzado de gasificador de biomasa/motor reciprocante para la generación electricidad. Tecnologia Química , 53-63 (2011)

Lisímaco Alonso, M. A. Estudio del secado artificial de la yuca en la Costa Atlántica de Colombia. En C. H. Ospina bernardo, sistemas modernos de producción, procesamiento, utilización y comercialización. (pág. 444 ). Cali. Colombia: CIAT (2000)

López, E. Caracterización y evaluación de la eficiencia térmica y de secado en un deshidratador híbrido (solar - gas). Tesis de maestría. Instituto politécnico nacional, Santa cruz Xoxocotlán, Oaxaca. (2011)

Machado, A. V. Estudio del Secado de Anacardo (Anacardium occidentale L.) mediante Secador Solar de Radiación Directa. . Información tecnologica, 21(1), 31-37 (2010)

Mahecha, J. Resultados obtenidos en deshidratación de uchuva. Seminario Internacional secado de productos agrícolas $2012,52-63(2012)$

Microsoft Office. Aplicación Solver excel. software Microsoft Office. Siñicon Valey: Microsoft Office (2007).

Motevali A, Minaei S. Effects of microwave pretreatment on the energy andexergy utilization in thin layer drying of sour pomegranate arils. www.ache.org.rs/CICEQ. Chem Industry Chem Eng Q.18:63-72, (2012)

Saavedra, J., Puente, L., y González, G. Automatización de un secador convectivo de aire caliente para fines de docencia en Ingeniería de Alimentos. Informatición Tecnologica, 19(14), 1-10 (2008)

Serrano, J., y Carranza, Y. Análisis teórico de la combustión en los quemadores de gas natural. Scientia Et Technica, 11(29), 139-143 (2000)

Treybal, R. Operaciones de Secado. Operaciones de transferencia de masa (págs. 723-748). Nueva York: Mc grawhill (1988) 